\title{
Diseño especulativo de segundo orden para una nueva "planeación" universitaria
}

\section{Second-order speculative design for a new university "planning"}

\author{
Daniel Lopera-Molano \\ Grupo MYSCO \\ Universidad de Ibagué \\ Ibagué, Colombia \\ daniel.lopera@unibague.edu.co
}

\author{
Hernán López-Garay \\ Grupo MYSCO \\ Universidad de Ibagué \\ Ibagué, Colombia \\ hernan.lopez@unibague.edu.co
}

\author{
Darío A. Martínez-Leal \\ Grupo MYSCO \\ Universidad de Ibagué \\ Ibagué, Colombia \\ dario.martínez@unibague.edu.co
}

\author{
Marly V. Tafur-Osorio \\ Grupo MYSCO \\ Universidad de Ibagué \\ Ibagué, Colombia \\ marly.tafur@unibague.edu.co
}

\author{
José J. González-López \\ Grupo MYSCO \\ Universidad de Ibagué \\ Ibagué, Colombia \\ Jair.gonzalez@unibague.edu.co
}

Resumen - En su libro el Cultivo de la Verdad, Fuenmayor [1] nos advierte que la presente época esta caracterizada por lo que, al parecer, es la mayor amenaza mortal para la institución occidental denominada universidad. Ello, menciona, se debe a un auge de la racionalidad instrumental, del reduccionismo y del pragmatismo ciego que endiosa al mercado y la tecnología. Lo anterior, arremete tanto interna como externamente contra la misión suprema de esta institución definida como la búsqueda crítica de la verdad. Paradójicamente, la misma institución universitaria está reforzando esa amenaza. En efecto, los sistemas de educación están demostrando su gran capacidad para reproducir las características de una sociedad instrumental, altamente especializada (y por ende fragmentada) y dominada por ese pragmatismo ciego que menciona Fuenmayor [1]. La estructura organizacional actual de las universidades y las prácticas que en ella se albergan han sido animadas por esa racionalidad que para poder controlar mejor divide y fragmenta incesantemente la realidad (es por ello que se multiplica el crecimiento disciplinar y el aumento de los programas especializados en las universidades). Pero a mayor refuerzo de los procesos de fragmentación, mayor la complejidad con la que hay que lidiar y mayor la incertidumbre y turbulencia que se genera. ¿Son conscientes de este fenómeno los entes universitarios encargados de planear y orientar el desenvolvimiento de nuestras instituciones?, pues cada vez menos pueden ayudarlas a navegar la incertidumbre y complejidad ya mencionadas al igual que, mucho menos, afrontar apropiadamente las amenazas contra la misión suprema de la Universidad. Con el ánimo de hacer una contribución a la comprensión y manejo de esa problemática desde la función de planeación universitaria, esbozaremos en el presente artículo una forma otra de "planeación". La propuesta se basa en lo que el teórico del diseño Tony Fry [2] denomina diseño ficcional de segundo orden. Dicho diseño hace posible, por una parte, el cobrar conciencia más clara de la naturaleza de la complejidad desbordante del presente. Y, por otra, facilita el comenzar a contribuir a la desfragmentación institucional situándonos críticamente en el presente y en el devenir histórico ontológico de la institución. En efecto, el mencionado diseño permite sembrar la semilla de una narrativa constitutiva de una comunidad 
educativa en la que dicha organización encuentre su identidad unitaria e identifique apuntadores emanados de la propia situación para dirigir su acción. En su primera fase, un proyecto piloto poniendo a prueba estas ideas fue iniciado en el 2020 junto con la Oficina de Planeación de la Universidad de Ibagué - Colombia. A partir del desarrollo de "estudios" de diseño virtuales, varios miembros de la comunidad universitaria se avocaron a construir narrativas especulativas de la institución que permitieron iniciar reflexiones holísticas de sí mismos y de la Universidad como un todo (análogo a una cibernética de segundo orden). En el presente artículo presentaremos algunos resultados preliminares de este proyecto piloto. Como conclusión parcial, urge el comenzar a sembrar una serie de prácticas organizacionales que permitan que estas formas otras de "planeación" puedan florecer en la comunidad universitaria, despejando caminos para el desarrollo de organizaciones viables, organizaciones que aprenden, organizaciones innovadoras y creativas capaces de reimaginarse, todas las cuales se requieren para poder navegar en las situaciones cada más complejas e impredecibles del mundo actual.

\section{Palabras clave- Planeación; universidad; diseño ficcional; neblina epocal; narrativización.}

Abstract - In his book the Cultivation of Truth, Fuenmayor [1] warns us that the present time is characterized by what, apparently, is the greatest mortal threat to the western institution called the university. This, he mentions, is due to a rise of instrumental rationality, reductionism and blind pragmatism that deifies the market and technology. The above attacks both internally and externally against the supreme mission of this institution defined as the critical search for truth. Paradoxically, the university itself is reinforcing that threat. Indeed, education systems are demonstrating their great capacity to reproduce the characteristics of an instrumental society, highly specialized (and therefore fragmented) and dominated by that blind pragmatism mentioned by Fuenmayor [1]. The current organizational structure of the universities and the practices that are housed in it have been animated by that rationality that in order to better control it divides and fragments reality incessantly (that is why disciplinary growth and the increase of specialized programs in the universities). But the greater the reinforcement of the fragmentation processes, the greater the complexity that must be dealt with and the greater the uncertainty and turbulence that is generated. Are the university entities in charge of planning and guiding the development of our institutions aware of this phenomenon? Because less and less can help them navigate the uncertainty and complexity already mentioned, as well as, much less, appropriately face the threats against the supreme mission. of the University. With the aim of making a contribution to the understanding and management of this problem from the university planning function, in this article we will outline another form of "planning". The proposal is based on what design theorist Tony Fry [2] calls second-order fictional design. This design makes it possible, on the one hand, to become more aware of the nature of the overwhelming complexity of the present. And, on the other, it makes it easier to begin to contribute to institutional defragmentation, critically situating ourselves in the present and in the ontological historical evolution of the institution. Indeed, the aforementioned design allows to sow the seed of a constitutive narrative of an educational community in which said organization finds its unitary identity and identifies pointers emanating from the situation itself to direct its action. In its first phase, a pilot project testing these ideas was started in 2020 together with the Planning Office of the University of Ibagué - Colombia. From the development of virtual design "studies", several members of the university community set about constructing speculative narratives of the institution that allowed initiating holistic reflections of themselves and of the University as a whole (analogous to second-order cybernetics ). In this article we will present some preliminary results of this pilot project. As a partial conclusion, it is urgent to start sowing a series of organizational practices that allow these other forms of "planning" to flourish in the university community, clearing paths for the development of viable organizations, learning organizations, innovative and creative organizations capable of reimagining yourself, all of which are required to navigate the increasingly complex and unpredictable situations in today's world.

Key Words- Planning; college; fictional design; epochal mist; narrativization

\section{Introducción}

El diseño actual del sistema de educación que predomina en Occidente, emerge de un complejo proceso de instauración de la Modernidad. El sociólogo Paul Goodman [3], hace más de medio siglo, destacó las características de sistema cerrado de los procesos de escolarización que refuerza acríticamente el patrón de vida dominante. Este sistema cada vez más pone la educación al servicio del mercado, "fabricando" e insertando "objetos" útiles a dicho sistema. Es por ello que Everret Reimer [4] afirmó que la escolarización perpetúa manifestaciones altamente insostenibles, tales como la aceptación de la dominación, el individualismo y el consumismo. Paralelamente, Iván Illich [5] lo caracterizó como una ritualización del progreso, en el que la educación entra al servicio del dios mercado y la tecnología que lo apoya. Conceptos anclados a la dinámica mercantil como "cliente", "servicio", "producto" y "proveedor" están siendo cada vez más usados en las universidades. Lo anterior, porque estas no sólo están al servicio del mercado, sino que se organizan como un producto que ofrece servicios educativos e investigativos al mejor postor y en competencia amplia con otras instituciones de educación superior. En ello, se va relegando a un segundo plano aquella misión originaria de formar buenos ciudadanos y cultivar la verdad para contribuir de manera significativa a la construcción de sociedades más justas y humanas.

Es claro entonces que la universidad ha venido perdiendo autonomía pues los fines para los que debe trabajar ya no son determinados por la propia institución sino por agentes externos a ella. Esto sucede, a pesar de que el elegante discurso estatal reconoce y apoya la autonomía universitaria $^{1}$. Sin embargo, mucho de este discurso se difumina y contrapone frente a los mismos criterios de validez de las naciones que promulgan las regulaciones de estándares de calidad, la dinámica de medición global 
sobre lo que se considera es conocimiento válido [6] y a la inserción en el lenguaje de palabras como "competencia", "privatización", "diversificación de oferta de productos", entre otras. Todo ello ratifica una pérdida de la esencia de la universidad frente a su constante búsqueda por la verdad, esa verdad plural, diversa, crítica y políticamente activa.

Sin embargo, lo mencionado es solo una manifestación que se sitúa en un contexto más amplio y que hace parte de una devastación sin precedentes, tanto fenoménica como ontológica. Ejemplo de lo primero es el exacerbado extractivismo y muerte de la madre tierra como ente vivo y vital para nuestra supervivencia. Con ello, también está el aumento de los niveles de los océanos, el calentamiento global, la perdida de los hábitats, las pandemias, entre muchas otras causas-consecuencias. Ejemplos de lo segundo están en la constante pérdida del sentido holístico de la existencia. Muestra de esta devastación ontológica radica en la fragmentación de la vida en todas sus formas: fragmentación del conocimiento, de la relación con la naturaleza, de nuestra relación con el cuerpo (fragmentado en mente, emociones y razón) y de las relaciones entre los seres humanos, por nombrar algunas. Fragmentación que favorece cada vez más el dominio de una racionalidad instrumental movida por una voluntad de poder y control. En Colombia, esta devastación se encarna en una historia de violencia, generada en buena medida por el despojo de futuros perpetrado contra millones de personas y por la imposición forzada de un solo modelo de vida y de una sola visión altamente reduccionista de las diversas formas de ser y estar en el mundo. En otras palabras, lo que en teorías decoloniales conocemos como la reproducción de un mundo hecho de un solo mundo, se plantea acríticamente como el proyecto dominante en nuestra sociedad. Los humanos perdemos, con esto, la práctica de innovar, crear otras posibilidades, construir un mundo en el que se desplieguen diversas maneras de ser y estar [7]. Así, se impide el florecimiento de Mundos otros, no necesariamente contrarios sino alternos, con los que es necesario dialogar para construir y hacer parte de caminos sustentables de vida.

Para Fry [8,9] la respuesta a esta situación es la del cultivo de un imperativo que nos convoque a todos, y que debe estar en el centro de nuestras relaciones, un imperativo hacia la construcción de un futuro sustentable. El lugar que Fry le asigna a la academia en esta misión le implica a esta no solo estar atenta a formar a las nuevas generaciones imbuidos de esta misión sino, sobre todo, ser crítica sobre sí misma y sobre aquello que reproduce. Esa doble tarea, de irse creando críticamente a sí misma y ser autorreflexiva en todos sus procesos de acople estructural con el sistema dominante, le sería esencial para poder lidiar con el presente. Más concretamente, es en la recuperación de prácticas ontológicas que estén ancladas a la construcción de autonomía, en tanto nos permitan diseñar un mundo en el que quepan muchos mundos [7] [10], que puede existir un posible camino de escape a la lógica dominante del modelo actual.

La actual situación global de pandemia, nos brinda una oportunidad para comenzar a correr el velo e ir tomando conciencia del modo de vida en que estamos atrapados el cual, por cierto, es generador de la pandemia y de muchas otras pandemias que se avecinan [11]. La devastación en la que nos encontramos no solo nos está exigiendo aprender a ver y lidiar con nuevas realidades sino también nos exige reimaginarnos, crear caminos hacia formas distintas, relacionales, críticas y auténticas de abordar la realidad. Pero esta acción institucional no podría ir separada de una acción comunitaria complementaria. En el campo de la salud, Chapela $[12,13]$ ubica a esta práctica de cuidado como terreno sin igual para desarrollar la capacidad en la que el ser humano corporeiza la posibilidad de diseñar, decidir y lograr futuros viables. Anotemos que, hablando de salud, en el marco de esta pandemia, un enfoque integral de la misma no pueda estar alejado de la idea de autonomía comunitaria y, en primera medida, de la capacidad de crear (diseñar) de las personas y de los colectivos. Por tanto, corresponde a las universidades regionales, como la Universidad de Ibagué, actuar en pro del desarrollo de la autonomía de las comunidades por medio del diseño de sus propios "trayectos" (frente al dominio de los "proyectos").

\section{La Planeación Universitaria}

¿Cómo se sitúa en el anterior contexto la planeación universitaria?

Sabemos que las organizaciones con jerarquías rígidas y formas usuales de tomar decisiones se han convertido en un obstáculo para sortear las situaciones complejas y rápidamente cambiantes del presente. Pero, en la medida en que la universidad se concibe más y más como una empresa que compite en el mercado de servicios educativos, esta rigidez se va flexibilizando para poderse adaptar mejor a los requerimientos del mercado de la educación. Así mismo, sus formas de planeación se orientan hacia el diseño de estrategias que les permitan a estas instituciones sobrevivir en mercados altamente competitivos [14]. Lo que exige sondear continuamente los comportamientos de escenarios futuros locales y globales para definir acordemente los objetivos, las políticas y las estrategias de mercado apropiadas.

Contrastando con estas formas de planeación y organización impulsadas por lo que podemos denominar una voluntad de mercado, tenemos las reformas universitarias puestas en marcha en las últimas décadas, como por ejemplo el Espacio Europeo de Educación Superior y el Espacio Europeo de Investigación, en el ámbito internacional, y la Estrategia Universidad 2015, en el nacional. Estas reformas parecieran estar promoviendo no una universidad centrada en el mercado sino una movida por los ideales de que la universidad debe ser un faro crítico de la sociedad y dar respuesta a las exigencias de la sociedad, tanto locales como globales [15]. Así, los marcos legales se orientan a catalizar diferentes formas de gobierno y planificación de las universidades, en función de las diferentes misiones, objetivos y estrategias que éstas quieran seguir; es decir, permitir que las universidades utilicen su autonomía para diferenciarse del resto y así poder atender mejor las necesidades locales de la sociedad en donde ellas se desempeñan [16,17]. Sin embargo, por otra parte, y alineada con la voluntad de mercado que impulsa a la universidad en la época presente, los entes gubernamentales que orientan la investigación y la docencia diseñan modelos de evaluación para financiar a las universidades con base a criterios en donde se desarrolla una dura competencia entre las universidades por fondos cada vez más escasos [18]. Estos factores provocan tensiones en las universidades cuyos perfiles no se correspondan con los estándares de los modelos 
subyacentes a esta visión de mercado, pues los estudios sobre productividad científica ponen de manifiesto que los resultados son diferentes en función del área de conocimiento. Además, aquellas universidades que tienen una importante carga docente e investigadora en el ámbito de las ciencias sociales y las humanidades tienen una clara desventaja $[19,20]$.

Por todo esto, y debido al incremento de la complejidad y al impredecible entorno local y global, se hace perentorio reorientar la planeación para atender estos retos y contribuir a la construcción de futuros sustentables para ellas y los entornos con los que trabajan.

\section{Metodología para una forma otra de "Planeación" Universitaria}

Acorde con todo lo anterior, en la Universidad de Ibagué se formó hace más de dos años un equipo de investigadores que empezaron a sentipensar formas otras de planeación. Este equipo encontró inicialmente inspiración en el trabajo del diseñador Tony Fry [8], quien preocupado por la crisis global y sus efectos desfuturizantes, como él les denomina, ha venido de larga data investigando y desarrollando herramientas para abordar la crisis. Consciente de que la misma requiere "salirse del cajón" y crear nuevas formas de pensar y reimaginar la situación que enfrentamos (so pena de reforzar la crisis misma, aunque creamos que estamos mejorando dicha situación) Fry ha propuesto el diseño ficcional y el diseño de futuros.

La aplicación de estas herramientas comprende cuatro fases articuladas y paralelas. Estas son: Comprensión sistémica del entorno o ambiente externo; Comprensión sistémica de la organización; Fines y búsqueda de Sentido organizacional y Navegación en turbulencia. Todos ellos orientados desde un enfoque coherente con la corriente del pensamiento sistémico de la Sistemología Interpretativa [1]. Expliquemos.

\subsection{Comprensión sistémica del entorno}

Toda situación compleja involucra a múltiples actores y perspectivas en juego. Esta fase requiere, bajo cada una de estas perspectivas, estructurar el entorno como un conjunto de elementos interconectados dinámicamente, de cuya interconexión emergen propiedades que ninguno de los elementos del conjunto por separado tiene. Estas estructuras no tienen carácter de ser modelos de la realidad sino dispositivos para ayudar a ganar una comprensión sistémica del entorno.

\subsection{Comprensión sistémica de la organización}

Vivimos imbuidos en nuestras formas de ser y hacer, por lo cual nos cuesta trabajo vernos a nosotros mismos. Una forma de "descubrirnos" es por contrastes (como cuando vamos a visitar otra cultura). El diseño ficcional, como práctica conscientemente cultivada en una institución, nos ayuda a lograr ese contraste.

\subsection{Fines y búsqueda de Sentido organizacional}

Igualmente, el diseño ficcional va más allá. Pues la creación de un mundo ficcional es en realidad un ejercicio recursivo de auto observación y reflexión crítica. Estos ejercicios son esenciales en entornos turbulentos y complejos, ya que en ellos es fundamental que en el seno de una institución se debata periódicamente sobre el sentido de lo que hacemos pues, en medio de la niebla de la turbulencia del entorno, no se trata de construir modelos del entorno para visualizar por dónde movilizarnos (cosa que ya dijimos no tiene mucho sentido) sino de, realmente, buscar el sentido de lo que somos y queremos ser, y orientarnos acordemente. La búsqueda de sentido de nuestro ser y hacer colectivo nos ayuda a encontrar claves en el presente para orientar colectivamente la acción institucional. Una forma sistémica de crear sentido es diseñando narrativas constitutivas de la identidad institucional. Expliquemos.

Debemos comenzar por entender que somos seres esencialmente narrativos . En el pasado, y en diferentes culturas, los seres humanos nos entendíamos narrativamente. Los grandes mitos fundacionales nos construían no como un "yo soy $\mathrm{X}$ y pertenezco a la comunidad Y". Sino como un somos colectivo histórico (por ejemplo, el pueblo elegido por Dios...etc.). Según Taylor [22], con el surgimiento del proyecto de la Modernidad hemos comenzado a construirnos como individuos desenraizados, descomprometidos, ahistóricos, individuos en sí mismos, separados completamente de su comunidad y de la naturaleza. Al concebirnos como individuos esencialmente separados de los otros y de lo otro, nuestra identidad, nuestro ser, no depende en absoluto de ellos. Es más fácil entonces cosificarlos y hacerlos instrumentos de nuestro propio proyecto de vida. Contrasta esta forma de entendernos hoy con las formas integrales como, por ejemplo, nos entendíamos en la Edad Media. Contrasta aún más cuando nos comparamos con culturas ancestrales como la de los $\mathrm{Kogui}^{2}$. En dicha cultura, cuando uno pretende nombrar, distinguir a una persona del colectivo, o de la naturaleza, el nombre lo fragmenta y desenraiza pues lo distingue como un ser aparte de uno y otra. El desconocimiento de este hecho social fundamental ha dado lugar a absurdos (cuando se ven desde la lógica Kogui) como el de empresas mineras pretendiendo comprarles la tierra o sacar licencias para explotar las montañas de la Línea Negra. Estas son acciones que atentan contra la esencia de su ser. Entendamos. No son Kogui, son Kogui-montañacielo-tierra... De igual manera, sus narrativas los constituyen integralmente lo que implica que no puedo entender la existencia de ninguno de ellos separado del mito constitutivo (narrativa madre) que le da sentido a lo que son.

El aprender entonces a re-narrativizarnos se vuelve una idea esencial para la búsqueda de sentido en tiempos de fragmentación epocal y búsqueda de sentido holístico. Y a esto ayuda el diseño ficcional, como veremos más adelante.

En su obra El Cultivo de la verdad, Fuenmayor [1] eleva este asunto de las narrativas a nivel histórico ontológico y le plantea un reto fundamental a la Universidad. Puesto en nuestros términos, el reto es el de asumir la misión histórica de ayudar a la humanidad a la recuperación de la capacidad de hacer sentido holístico de la existencia. En los términos de Fuenmayor [1]: La

\footnotetext{
${ }^{2}$ Los Kogui son un pueblo originario de Colombia que habita en la Sierra Nevada de Santa Marta. Tienen su propio idioma, perteneciente a la familia Tayronas.
} 
misión fundamental epocal [de la universidad] es la de la reconstrucción de la historia ontológica regresiva de Occidente de manera que dé cuenta de nuestro presente. Ello implicaría la gran tarea universitaria, a nivel general, de llevar a cabo la reconstrucción "arqueológica" de una narrativa histórico-ontológica general que constituiría el hilo conductor de una nueva mitología, sobre la base de la cual, si llegase a convertirse en mitología viva, operaría la reapropiación de nosotros mismos [es decir, la recuperación de la capacidad de hacer sentido holístico de la existencia]. A nivel particular, esta reconstrucción tendría que llevarse a cabo en las variadas disciplinas y áreas del saber. Con esto, Fuenmayor [1] quiere decir que todo el trabajo de investigación, desarrollo y enseñanza que se lleva a cabo normalmente en una universidad, tendría que estar provisto de su sentido históricoontológico. "Es decir, debe estar fundado en una profunda consciencia de cómo llegó a ser lo que es en términos histórico ontológicos" (pág.221), nos referimos a la incorporación del devenir histórico-ontológico en la vida de los juegos lingüísticos de las disciplinas - “así como la mitología está incorporada en los juegos lingüísticos de las culturas "saludables" (p. 222, énfasis añadido).

Como vemos, no se trata de construir simplemente historias narrativas (y mucho menos historiografías) de una institución. Las narrativas constitutivas de que hablamos deben servir de fundamento para dar cuenta de cómo llegamos a ser lo que hoy somos y cómo llegó a constituirse nuestro actual modo de relacionarlos con la realidad. Una narrativa constitutiva de una comunidad es el fondo que hace posible la forma de cualquier cosa o hecho, que acaece, que se presenta en esa comunidad. Por ejemplo, hoy día todo lo que se presenta se nos muestra como un dispositivo siempre listo para ser usado [23]. Por supuesto, en cada caso particular habrá que ver como se encarna este fondo-forma. Y esto es lo que tiene que hacer una narrativa constitutiva. Hablaremos más al respecto cuando toquemos el tema de la narrativización y el diseño ficcional especulativo.

\subsection{Navegación en turbulencia}

La narrativa constitutiva de una institución le suministra un fondo sobre el cual se yergue en general su sentido organizacional. Esto permite entonarse con el presente y buscar claves orientadoras específicas en la realidad en que estemos inmersos, que fluye continuamente y se va manifestando día a día en toda su complejidad. En el paradigma tradicional de planeación queremos imponer sobre la realidad una orientación acorde con los fines y búsquedas organizacionales estratégicamente pensados y planeados. Pero desde esta forma otra de planeación que estamos bosquejando es la realidad de la situación la que, con una escucha atenta (que tiene por fondo la narrativa constitutiva), da claves orientadoras.

Como veremos más adelante, las anteriores cuatro fases se orientan sobre todo a crear condiciones de posibilidad para desarrollar no sólo la práctica de narrativizar (práctica clave para la búsqueda de sentido organizacional) sino también la de especular y auto reflexionar.

\section{Diseño Ficcional de segundo orden, y las prácticas de la auto observación, narrativización y especulación}

Seguidamente, vamos a introducir el diseño ficcional de segundo orden y mostrar la conexión del mismo con lo que llamaremos las prácticas de la auto observación, narrativización y especulación. Sembrar dichas prácticas en los niveles organizativos de planeación de una institución universitaria será nuestra respuesta a la necesidad de crear formas otras de planeación para poder atender la crisis del presente.

Fry [2] define el diseño ficcional de segundo orden de la siguiente forma:

El término "ficciones de diseño de segundo orden" se basa en el pensamiento y el uso del término cibernética de segundo orden. Como tal, la producción de la ficción es un ejercicio recursivo de auto observación crítica y reflexión que devuelve lo que se crea a su autor, como una observación de sí mismo del acto y del objeto de la creación (p.4, énfasis añadido).

Hagamos notar, primero que todo, que un objetivo primordial del diseño ficcional de segundo orden (cuando lo aplicamos a una organización) es estimular en una organización el desarrollo de una práctica de auto observación crítica y reflexiva sobre la misma y el presente en el que se encuentra situada.

La noción de crítica que hemos adoptado en nuestro articulo tiene inspiración kantiana: conducir una crítica de $\mathrm{X}$ es revelar aquello que condiciona $\mathrm{y}$ hace posible $\mathrm{X}$. Por tanto, una autobservación critica busca revelar justamente el fondo que nos condiciona y hace posible que seamos lo que somos. Una narrativa constitutiva de identidad institucional daría cuenta de cómo llegamos a ser lo que hoy somos (institucionalmente hablando). Es decir, daría cuenta de cómo hemos llegado a darle el particular sentido que le damos al mundo, a lo que somos y a lo que hacemos, por contraste a cómo le dábamos sentido anteriormente ${ }^{3}$.

Completaría este cuadro el ejercicio especulativo que narrativamente buscaría construir un espacio de posibilidades de ser mundos otros (no ficticios como las de la ciencia ficción), de construirnos de otra manera, tejiendo un fondo en el que el modo en que las cosas se presenten tengan un cariz de no real aún pero, definitivamente, no meramente ficticio.

4.1 Algunas consideraciones acerca de los métodos de creación de un diseño ficcional de segundo orden.

Al respecto Fry [2] nos dice que los métodos empleados para la creación de un diseño ficcional de segundo orden tienen que estar relacionadas con su particularidad situada. Es decir, con el contexto especifico y particular foco de atención del diseño. No obstante, hay ciertos aspectos generales metodológicos que deben considerarse cuando abordamos la tarea de diseñar una ficción. Algunos de estos aspectos a considerar son:

naturaleza y hasta los principios morales, dentro de un gran complejo instrumental regulado por la tecnología y el mercado. Pero en la época precedente es el Progreso de la Razón la idea fuerza que domina el modo de aparecer las cosas. 
- Entorno, tiempo y ubicación: para ser comprensible y evaluada adecuadamente, una ficción de diseño de segundo orden debe verse como algo que surge [del contexto específico]; está influenciada y situada funcionalmente en relación con las fuerzas y condiciones de su contexto en sus condiciones temporales de intercambio y de relacionalidad con la que actúa.

-Todas las cosas creadas salen de un contexto cultural y se dirigen hacia una orientación cultural: todo lo que da voz, representa visualmente y anima la ficción necesaria para expresarlo claramente.

-Condiciones de limitación: para contrarrestar la propensión utópica de las ficciones de diseño, se deben establecer, reconocer y responder a las condiciones de limitación.

\subsection{Ejemplo de diseño ficcional}

A continuación, daremos un ejemplo de diseño ficcional ${ }^{4}$. Se trata de una comunidad colombiana en la que se está pensando desatar un proceso de autoobservación crítica y reflexión/acción a través de un diseño ficcional de segundo orden. La idea es que este diseño sirva de semilla para que este proceso pueda crecer orgánicamente en la comunidad, y conduzca a una serie de prácticas transformadoras de dicha comunidad, guiadas por un sentido cada vez más claro de su identidad y de cómo llegó a ser lo que es en el presente. La ficción que se diseña es una narrativa que cuenta cómo una maestra de una escuela local se inspira en una historia que ha leído en una vieja revista acerca de cómo en un pequeño pueblo egipcio sus habitantes lograron transformarlo en un centro importante de cerámica artesanal. Inspirada en esa historia la maestra comienza a tomar una serie de acciones, en las que poco a poco va involucrando a distintos miembros del pueblo, quienes activamente tejen la visión de un centro artesanal que se va convirtiendo no sólo en un motor de desarrollo económico de la comunidad sino en un Centro de Formación Integral de la Región, donde las comunidades aprenden a cultivar prácticas de autonomía y cuidado de todos y cada uno de sus miembros y de la madre naturaleza ${ }^{5}$.

4.3. Tres métodos-prácticas de diseño ficcional de segundo orden

Inspirados en los anteriores lineamientos, nuestro grupo de investigación ha venido bosquejando tres métodos-prácticas, así llamados porque, si bien es cierto que un trabajo de diseño ficcional se puede catalizar inicialmente con los tres métodos que expondremos a continuación, no obstante lo que realmente se cataliza en la organización es un proceso de desarrollo de prácticas que, como las cinco disciplinas que habla Peter Senge, hay que incorporar a la vida cotidiana de la organización o comunidad. En nuestro caso específico serían prácticas que habilitarían los organismos de planeación de la universidad con las "disciplinas" necesarias para comenzar a enfrentar, con formas otras de planeación, los entornos complejos y turbulentos que caracterizan a la época presente.
4.3.1. La narrativización: Llamamos narrativizar a la práctica constante que brinda coherencia narrativa, y por tanto unitaria, a la vida. Se trata, como en el ejemplo de la sección 4.2., de crear narrativas ficcionales que, por contraste con el presente, induzcan en una comunidad $u$ organización una búsqueda de sentido del presente y de cómo llegó a ser lo que es. Es decir, a darle el particular sentido al mundo y a lo que acontece (por contraste a cómo le daba sentido a su quehacer y ser anteriormente).

4.3.2. La especulación: En nuestro trabajo con los entes de planeación de la universidad, la herramienta de la especulación resulta ser un complemento a la de narrativización. Comprendemos la especulación, por un lado, como la generación de suposiciones, reflexiones o ideas sobre algo específico. En el caso del Diseño especulativo, este ejercicio siempre tiene una cierta orientación al futuro y requiere de gran capacidad tanto creativa como relacional. Es por esto que la práctica de la especulación, desde la perspectiva del diseño de futuros [9], implica poder articular ideas y suposiciones coherentemente como una narrativa integral de pasado, presente y futuro. Fry lo menciona en términos de diseñar desde el futuro hacia el presente sin perder la posibilidad de explorar ideas que al inicio suenen extrañas, o poco convencionales. Pero, desde la marcha dialógica de la metodología se van transformando orgánicamente.

4.3.3. La autorreflexión: Esta práctica es característica de los procesos de segundo orden, en los que el observador se pregunta por cómo está observando. Desde una relación triádica con los otros dos términos, mientras el observador desarrolla la práctica de narrativizar podría también preguntarse por qué futuros está favoreciendo $\mathrm{y}$, al hacerlo, darse cuenta del lugar desde el cual se ubica para especular. A propósito, al término de especulación también puede derivársele, en su raíz latina, la connotación de espejo o reflejo. Esto hace que lo especular también permita y favorezca la autorreflexión y la búsqueda de sentido integral.

La práctica constante de explorar esos mundos, poblados por artefactos ficcionales, nos reta a vivirlos y comprenderlos. Pero, sobre todo, nos reta a contrastarlos con el presente que vivimos. Las ficciones diseñadas para darle vida a ese mundo ficcional son traídas al aquí y ahora de nuestro mundo presente, con el propósito no de ver como los copiamos/implantamos en el presente sino para cuestionarlo y desatar debates y reflexión crítica acerca del presente que vivimos. Lo anterior, ayuda a revelar cosas que están sucediendo justamente en este momento, en frente de nosotros, pero que nunca nos dimos cuenta, ni asumimos la perspectiva adecuada para verlas

5. Diseño ficcional y la búsqueda de formas otras de planeación en la Universidad de Ibagué

De lo anterior se colige que narrativizar, especular y auto reflexionar son la tríada de una misma práctica integral. Para poder llevarla a cabo en el contexto de la Universidad de Ibagué - Colombia, fue necesario comprender cómo se estaba percibiendo la Universidad a la luz de una primera narrativa inicial. Esta fue la primera

de tiendas artesanales del pueblo, mesas de venta objetos [Nosotros añadimos un sexto elemento que se refiere al cierre de la narración en un tono de tensión dilemática: Una comunidad que es absorbida por las reglas del mercado y la globalización versus una comunidad en la que el Centro Artesanal se transforma en Centro de Formación Integral y motor de desarrollo autónomo de la región.

\footnotetext{
${ }^{4}$ El ejemplo está inspirado en un diseño ficcional planteado por Fry [2].

${ }^{5}$ Fry [2] plantea una serie de sugerencias básicas acerca de la estructura de la narrativa. 1. Escriba la apertura de la historia. 2. Seleccione un pueblito y fotografielo 3. Arregle las fotos usando Photoshop para que el pueblo luzca recién pintado. 4. Seleccione uno o más tipos de artesanías. 5. Invente personajes, avisos
} 
pregunta del ejercicio iniciado en 2020, en su primera fase, junto con la Oficina de Planeación de la Universidad de Ibagué, a partir del desarrollo de estudios virtuales de diseño desde los cuales varios miembros de la comunidad le dieron forma y sentido integral a la narrativa inicial de una universidad necesaria y posible para la época.

Metodológicamente se consideró oportuno abordar estos ejercicios creativos a partir de la generación de escenarios especulativos, desde los cuales pudiésemos iniciar conversaciones sobre posibilidades y búsqueda de sentido integral de nuestro quehacer universitario ante la crisis del presente. Como en el ejemplo de la sección 4.2, se diseñó inicialmente una narrativa semilla que desatara en los participantes de los estudios virtuales especulaciones acerca de una posible Unibagué Reimaginada. Esta narrativa semilla fue diseñada por el equipo de coinvestigadores como una historia desafiante para los participantes y a la cual se les invitaba, más que a responder, a continuar narrándola. Claramente, la narrativa tenía que ser abierta y plantearle retos desafiantes a la imaginación. El reto se caracterizó por buscar que los participantes no recurrieran a respuestas cotidianas pues era evidente en la narrativa que éstas no podrían funcionar.

En suma, acorde con el ejemplo de la sección 4.2. diseñamos la narrativa semilla y la sembramos invitando a los participantes a continuar la historia narrándola con el hacer-imaginar. Y narrándola también se fueron narrando y descubriendo maneras de interpretar holísticamente su devenir hasta el presente.

\subsection{Los estudios virtuales de diseño}

Para comenzar a poner a prueba estas ideas del diseño ficcional $\mathrm{y}$, particularmente, ir sembrado en la Universidad las tres prácticas antes mencionadas, se diseñaron durante el segundo semestre de 2020 cinco encuentros en el formato de "estudios virtuales de diseño" usando la plataforma de Google Meet. Cada encuentro tuvo 5 participantes entre directivos, docentes y demás funcionarios de la Universidad, un moderador y un relator. Las relatorías se desarrollaron en un tablero virtual de libre acceso, que permitió realizar esquemas mentales e imágenes enriquecidas con gran facilidad.

\section{Resultados}

Los resultados iniciales de este ejercicio mostraron que los participantes efectivamente pudieron construir una narrativa base de universidad en proceso de transformación. A este nivel inicial se buscaba tan sólo poder desplegar narrativamente una primera imagen de cómo los participantes ven la Universidad en el presente, su entorno local y global. Seguidamente, y usando como contexto la narrativa base iniciamos una serie de ejercicios especulativos que buscaban desencajar (sacar de la caja al pensamiento y liberar la imaginación) esas percepciones iniciales para así poder ganar una perspectiva más amplia de la situación de la Universidad. Una, desde la cual pudiera dibujarse críticamente la manera como se percibe la situación presente y así favorecer la pregunta por ¿cómo llegamos a ser esto que somos? Esta pregunta es clave para desplegar una búsqueda de sentido holístico. Y como ya lo mencionamos anteriormente, la complejidad y turbulencia del presente requiere no del diseño de planes (es decir plantas, como la planta de un edificio a ser construido, siguiendo la etimología francesa de la palabra
"Plan") sino del diseño de contextos interpretativos que nos permitan estructurar un sentido holístico de lo que somos y lo que hacemos. No planificar sino interpretar.

Los ejercicios de desencajamiento usando el diseño especulativo condujeron a Diseños especulativos de narrativas enmarcadas en la idea de "redes", "sistemas" o, incluso, "cuerpos" u "organismos" como respuesta a la necesidad de articulación, relacionamiento y pertinencia regional y global de la Universidad. La conexión narrativa que construyeron los participantes para que esta idea central fuera posible en la narración fue a partir de plantear una "cultura organizacional" caracterizada por ser "flexible", "fluida" y "orgánica" en pervivencia con la tecnología, en reconocimiento de lo "diverso" y "pertinente"; ofreciendo condiciones para un "currículo feliz", para la "creación", la "diversión" y la "reflexiónacción". Como consecuencia de estas condiciones, los participantes mencionaron que se hace posible diversificar la oferta académica en distintos niveles, desde escenarios no convencionales, y respondiendo a los cambios de época $\mathrm{y}$ de paradigmas que estamos viviendo.

Cuando se orientaron las conversaciones sobre el objetivo que perseguían en esa narrativa que construyeron, se mencionó recurrentemente que era "construir región", lo cual implicaba la transformación de los territorios desde el "cuidado de sí, de lo otro y los otros". Así, esas relaciones narrativizadas no solo tenían un fin relativo a formar profesionales sino, sobre todo, a "cuidar" integralmente. Lo anterior, evidencia el recuerdo de un sentido esencial de la idea de universidad haciéndose presente en una narrativa especulativa, pero con total conexión con los deseos e intereses de los participantes. Así, se presenta también como un posible ejercicio de conexión teleológica para el cual habrá que explorar más a fondo su aplicación en las transformaciones concretas y recurrentes de la institución.

\section{Conclusiones y Reflexiones Para Futuras Aplicaciones}

Hallazgos parciales de nuestra investigación muestran que los grupos que participaron en los estudios virtuales experimentaron una gran diferencia entre los ejercicios de diseñar planes y los de diseñar contextos interpretativos que orienten la búsqueda de sentido. En el paradigma de la planeación a que ellos estaban acostumbrados, los ejercicios de planeación que se realizaban en la Universidad iban orientados a identificar las funciones de las unidades, objetivos y metas, y a dibujar escenarios pesimistas y optimistas para poder decidir cómo actuar en los entornos complejos que ya mencionamos. Pero, con la metodología de diseño ficcional usada en los estudios virtuales reportados, los grupos comenzaron a comprender (aunque es temprano para decir si también a asimilar) que la tarea de orientar el devenir de una institución se ancla a narrativizaciones, especulaciones y auto observaciones que permiten ganar una visión integral de la organización en relación con el mundo y a desplegar los sentidos que pueden orientarla en la neblina turbulenta del presente.

En cuanto a comenzar a asimilar la práctica de la narrativización encontramos que fue central diseñar las semillas narrativas con finales abiertos y retos e, incluso, paradojas. Ello dispuso a los participantes a un ambiente propositivo, pero siempre anclado a lo viable y factible. Igualmente, encontramos que el diseño de la narrativa 
debe poder hacer conexión con los contextos de experiencia de los participantes e invitar al diálogo. Es por eso que al plantear contextos ficcionales es importante que los participantes se comprendan insertos en ellos, al igual que establezcan con claridad cuál es la estructura esencial que no se puede traspasar, de lo contrario, deja de estar inserto en esa narrativa. Por otra parte, si la narrativa en relación con el contexto de experiencia de los participantes limita y restringe el campo de juego ficcional de los mismos, entonces, se puede perder en el grupo la posibilidad creativa para el diálogo de saberes, de la cual emergerá un sentido holístico para la acción humana.

Con relación a las prácticas de auto observación, que las narrativas co-construidas por el grupo deben favorecer, no fue posible ejercitarla en el corto tiempo de los cinco estudios que se programaron en el 2020. Suponemos que, dada la continuación cíclica ascendente de las mismas, eso podrá permitir que los participantes vuelvan a revisarlas. De continuar este estudio en el 2021 es claro que tendremos que comenzar a enfocarnos en las estrategias que permitan cultivar un ethos organizacional en el que las tres prácticas se vayan arraigando ${ }^{6}$.

Para finalizar, el gran reto que tenemos por delante es el de lograr ir desarrollando en la Universidad las condiciones de posibilidad para que una narrativa colectiva se vaya tejiendo; una narrativa cuya gran misión tendría que ver con el carácter regional de la Universidad y su compromiso con el desarrollo de la región. Se trataría de la recuperación del tejido vital de la región. Dicho tejido no es otro que la urdimbre narrativa de las cosmovisiones y saberes relacionales, el cual serviría de base firme a la sustentabilidad de las comunidades.

En suma, este gran reto nos permite comenzar a vislumbrar que la orientación de nuestras instituciones tendrá cada vez menos que ver con planeadores y más con tejedores de narrativas, vía prácticas de narrativización, especulación y auto reflexión.

\section{Agradecimientos}

Especial reconocimiento a los 25 participantes de los estudios virtuales de diseño y su intención de continuar el proceso narrativo que les propusimos. Igualmente, a nuestros colegas Tony Fry y Arturo Escobar por sus importantes aportes conceptuales y metodológicos desde los cuales nos estamos ubicando para este ejercicio de Diseño ficcional especulativo de segundo orden en la Universidad de Ibagué, Colombia. A las directivas institucionales por acoger la idea y considerarla como opción válida frente al entorno turbulento e incierto en el que nos encontramos.

\section{REFERENCIAS}

[1] R. Fuenmayor, "El cultivo de la verdad: la esencia de la universidad," Ediciones Unibagué, 2016.
[2] T. Fry, "Design Fictions - Colombia", en Seminario virtual Universidad de Ibagué, 18 agosto 2020 (https://drive.google.com/file/d/1zNvKWfaimxqOqCWIbwDTw gTV14VLQNNs/view? usp=sharing)

[3] P. Goodman, "Compulsory miseducation," New York: Horizon Press, 1964.

[4] E. Reimer, "La escuela ha muerto. Alternativas en materia de educación", Barral, 1974.

[5] I. Illich, "Schooling: The Ritual of Progress," in New York Review of Books, 1970.

[6] B. L. Hall, and R. Tandon, "Decolonization of knowledge, epistemicide, participatory research and higher education," en Research for All, vol. 1-1, pp. 6-19, 2017. (DOI 10.18546/RFA.01.1.02)

[7] A. Escobar, "Autonomía y diseño: la realización de lo comunal," Editorial Tinta limón, 2017.

[8] T. Fry, "A new design philosophy: an introduction to defuturing," UNSW Press, 1999.

[9] T. Fry, "Design futuring" Berg Publishers, 2010.

[10] S. Marcos, y C. C. EZLN, "Cuarta Declaración de la Selva Lacandona," en Utopías, nuestra bandera: revista de debate político, vol. 167, pp. 125-133, 1996.

[11] P. Descola, "El modelo de desarrollo capitalista es una especie de virus para nuestro planeta," en BBC News, 23 junio 2020.

[12] M. C. Chapela, y A. Cerda, "Investigación cualitativa sanadora. Por los caminos de la investigación cualitativa sanadora," en Exploraciones narrativas y reflexiones en el ámbito de la salud. Universidad Autónoma Metropolitana, unidad Xochimilco, pp. 120-138, 2010

[13] M. C. Chapela, "Entre poética y didáctica: promoción de la salud orientada por una utopía emancipadora (PSE)," en Entre poética y didáctica. Narrativas en el campo de la salud, pp. 43-70, 2015.

[14] J. M. Bryson and W. R. Roering, "Strategic planning options for the public sector," Handbook of public administration 2, 1996.

[15] MICINN, Ministerio de Ciencia e Innovación, "Borrador de la Estrategia Universidad 2015," en La gobernanza de la universidad y sus entidades de investigación e innovación, Fundación CyD y CRUE, 2010. (http://firgoa.usc.es/drupal/files/ Documento\%20 Gobernanza\%20CRUE\%20FCYD.pdf)

[16] European Commission, "European Universities: Enhancing Europe's Research Base," in Final report by the Forum on University-based Research. Luxembourg, Publications Office of the European Union, 2005.

[17] V. Pérez Díaz, "La crisis endémica de la Universidad española," en Claves de razón práctica, vol. 158, pp. 38-43, 2005.

[18] M. A. Quintanilla, "La investigación en la sociedad del conocimiento," en Revista Iberoamericana de Ciencia, Tecnología y Sociedad, vol. 8-3, pp. 183-194, 2007.

[19] G. Buela-Casal, M. P. Bermúdez, J. C. Sierra, R. Quevedo-Blasco, y A. Castro, "Ranking de 2009 en investigación de las universidades públicas españolas," en Psicothema, vol. 22, pp. 171-179, Diciembre 2010.

[20] J. Olmo Peñuelas, E. Castro Martínez, and L. Manjarrés Henríquez, "Knowledge Transfer in Humanities and Social Science Reserch Groups: The Influence of Organizational Factors," en Social Sciences Abroad, vol. 3, pp. 99-108, 2011.

[21] A. MacIntyre, "Tras la virtud," Crítica, 1987.

[22] C. Taylor, "Sources of the Self," Harvard University Press, 1989.

[23] M. Heidegger, "La pregunta por la técnica," en Revista de filosofía, vol. 5-1, pp. 55-79, 1958.

\footnotetext{
${ }^{6}$ Sospechamos que la lógica de este arraigo debe compartir algunas ideas con la Learning) ha recopilado en relación con la siembra de las cinco disciplinas amplia experiencia que por ejemplo la fundación SOL (Society for Organizational 